\title{
Contingent Existence and the Reduction of Modality to Essence
}

\author{
Trevor Teitel \\ New York University \\ trevor.teitel@nyu.edu
}

This paper first argues that we can bring out a tension between the following three popular doctrines: (i) the canonical reduction of metaphysical modality to essence, due to Fine, (ii) contingentism, which says that possibly something could have failed to be something, and (iii) the doctrine that metaphysical modality obeys the modal logic S5. After presenting two such arguments (one from the theorems of S4 and another from the theorems of B), I turn to exploring various conclusions we might draw in light of these results, and argue that none comes cost-free. In the course of laying out possible responses to my arguments, we'll have a chance to evaluate various doctrines about the interplay between contingency and essence, as well as develop some alternative reductions of metaphysical modality to essence. I don't come down decisively in favour of one response over the others, though I say some things that point towards the conclusion that essence has no role to play in reducing metaphysical modality.

\section{Introduction}

My goal in this paper is to bring out a tension for proponents of reducing metaphysical modality to essence, and to explore various morals we might take away from this result. Roughly for now, our tension will arise from the following three popular doctrines:

- S5: Metaphysical modality obeys the modal logic S5.

- Contingentism: Possibly something could have failed to exist (where throughout the paper unqualified uses of modal locutions, including ' $\square$ ' and ' $\diamond$ ', should be understood as expressing metaphysical modality).

- Modality to Essence: Metaphysical modality reduces to essence in the canonical way, due to Fine (1994)—we'll spell out the reduction below.

One response to our result is to hold onto the canonical understanding of the reduction to essence, and so view our discussion as an 
argument either against $\mathbf{S}_{5}$ or against Contingentism. Either way, we'd have an interesting upshot: proponents of the canonical reduction to essence must adopt a non-standard view about the logic of metaphysical modality, or else they are committed to necessitism, the doctrine that necessarily everything necessarily exists (the negation of contingentism). A second line of response, which we'll spend the most time with, is to reject the canonical understanding of the reduction to essence, but to try to come up with some modified version of the reduction that is consistent with $\mathrm{S}_{5}$ and Contingentism (throughout we'll use 'canonical reduction' to describe the version of the reduction to essence presented in Fine 1994, which is the version that is almost universally under discussion in the literature). We'll look at two alternative reductions of modality to essence that might do the trick. Even those already inclined to reject $\boldsymbol{S}_{5}$ or Contingentism should have something to gain from this discussion, which bears on the project of reducing modality to essence more generally, even setting aside our arguments. A third line of response would be to hold onto $\mathrm{S}_{5}$ and Contingentism yet reject any modifications of the canonical reduction to essence, and so view our discussion as an argument against any kind of reduction of modality to essence. The alternatives to the canonical reduction that we'll see all have problems of their own, so this third line of response has some plausibility. Finally, we'll see other lines of response stemming from the different assumptions we'll need in our arguments, which point to some controversial new theses about what's essential to what.

Here my main aim is to bring out the tension between our initial doctrines and evaluate the possible replies, rather than try to decisively come down in favour of one reply over the others, though I'll say some things that point towards the strong conclusion that essence has no role to play in reducing metaphysical modality. Nonetheless, the brief sketch we just gave of some of the different replies to our arguments already shows that no reply comes cost-free, and so our arguments have interesting upshots no matter which way we go.

We'll get to our arguments in \$3. First, let's spell out exactly what the three initial doctrines say.

\section{The three doctrines}

Starting with $S_{5}$, the two axiom schemas of $S_{5}$ we'll show to be in tension with Contingentism and Modality to Essence are the 4 
schema, that what's necessary is necessarily necessary, and the B schema, that what's the case is necessarily possible. Given that the logics $\mathrm{S}_{4}$ and $\mathrm{B}$ each suffice to bring out the tension, our argument in fact employs only the strictly weaker assumption that metaphysical modality obeys either the modal logic $\mathrm{S}_{4}$ or the modal logic B. Still, it will be illustrative to see how the tension arises for both $\mathrm{S}_{4}$ and $\mathrm{B}$, each of which brings out different issues. So we'll present two separate arguments, one from the theorems of $\mathrm{S}_{4}$ and another from the theorems of B, which strengthens our claim that there's a real tension here. The theorems of $\mathrm{S}_{5}$ are widely regarded as non-negotiable constraints governing metaphysical modality. Moreover, the main objections to $\mathrm{S}_{5}$ target only instances of the 4 schema (and its necessitations) as genuine constraints, and these objections don't extend to the theorems of B. So advocates of these objections at best avoid our argument from the theorems of $\mathrm{S}_{4}$, but still must address our argument from the theorems of B. ${ }^{1}$

I'll use universal quantification into sentence position to express what it means for metaphysical modality to obey some constraint embodied in some modal schema or logic. For example, we'll say that metaphysical modality 'obeys' or 'is governed by' the Tschema, that what's necessary is the case, if and only if $\forall p(\square p \rightarrow p)$, where this latter formula, with its sentential quantifier, is part of the language in which we're conducting our discussion, not the propositional or first-order modal languages usually under discussion when presenting the familiar modal logics. (For the general method for moving from some theorem of some logic to the relevant universally quantified statement, and some rationale for this method, see the

\footnotetext{
${ }^{1}$ The main objections to $S_{5}$ alluded to in the main text stem from the 'modal sorites' arguments against the 4 schema as a constraint governing metaphysical modality, due to Chandler (1976) and further elaborated by Salmon (1981, pp. 229-52; 1989). But these arguments remain controversial, and most still subscribe to $S_{5}$. For a sampling of some attempts to respond to these arguments, see Williamson (1990, \$8.3), Hawthorne (2006, p. 241 n.8), Leslie (2011), and Murray and Wilson (2012). Another potential source of resistance to S5 stems from counterpart theory, which some, including Lewis (1968, pp. 123-4), take to show that neither the 4 schema nor the B schema embodies a constraint governing metaphysical modality. (Thanks to an anonymous referee for this suggestion.) However, (i) counterpart theorists disagree as to whether their view, properly understood, carries these revisionary logical consequences, (ii) on some ways of implementing counterpart theory, the programme is committed to a reduction of modality in terms of first-order quantification over some additional ontology, and so won't offer solace to proponents of the canonical reduction to essence looking for a way to hold onto their view in light of our arguments, and (iii) counterpart theory remains a controversial programme that has been criticized on several fronts. See Dorr (MS) for extensive discussion of these three issues and an evaluation of some of the problems facing counterpart theory.
} 
discussion of 'metaphysical universality' in Williamson 2013, ch. 3.) We could have stuck with schematic statements of various modal principles throughout, using only particular instances to derive our tension (and those who prefer to do so won't have trouble recasting our discussion in this mode), but universal quantification into sentence position captures the generality implicit when we claim that metaphysical modality obeys or is governed by some schema or logic, and allows us to bring out our tension in full generality without being bound by the expressive resources of any particular language. There's a vexed issue about how to interpret higher-order quantification (where quantification into sentence position is just o-adic higher-order quantification), whether in terms of substitutional quantification relative to some idealized language, as restricted first-order quantification over some special kind of objects (such as properties, propositions or sets), or as an irreducible form of quantification whose interpretation can be given only in a metalanguage that itself employs higher-order resources. We can stay neutral on this issue, which is orthogonal to the tension I'm bringing out here (though I've set aside interpretations in terms of plural quantification, which arguably can't do justice to quantification into sentence position or to how higher-order quantifiers interact with modal operators). For convenience I'll generally write as if quantification into sentence position is just first-order quantification over propositions, which simplifies some of the exposition in what follows, though nominalists who don't think there are any propositions can paraphrase away 'proposition' talk using higher-order quantifiers and interpreting such quantifiers using one of the alternatives to the first-order interpretation (the same is true of our use of 'property' talk later on). ${ }^{2}$

Contingentism is the negation of necessitism, the doctrine that necessarily everything necessarily exists (for a defence of necessitism see especially Williamson 2013). You might think that necessitism is obviously false; for instance, you might think, 'Surely it's possible that my parents never met, in which case I wouldn't have been born and so wouldn't have existed'. These sorts of worries aren't decisive, given the sense of 'existence' at issue in the debate between necessitists and contingentists. For Williamson, ordinary uses of 'existence' generally express properties like being concrete, or being located in spacetime,

\footnotetext{
${ }^{2}$ See Williamson (2013, pp. 235-61) for a survey of some of these different options for interpreting higher-order quantification, citations to relevant literature, and a defence of the view that we shouldn't try to reduce higher-order quantification to anything else.
} 
and Williamson is happy to grant that having these properties is a contingent matter. But Williamson's necessitism is a doctrine about what he calls the 'logician's sense of existence', according to which ' $x$ exists' $=_{d f} \exists y(x=y)$, where here the quantifier is absolutely unrestricted. We'll follow Williamson from now on in using 'is something' and 'has being' interchangeably to express this notion, which will ensure that it doesn't get conflated with more ordinary uses of 'exists'. Williamson's diagnosis of the worry a few sentences back is that the objection conflates these different senses of 'existence': although if you hadn't been born you wouldn't have been concrete or located in spacetime, for necessitists you would still have been something, a contingently non-concrete entity. So we see that necessitism isn't immediately refuted; nonetheless, the doctrine is hotly contested, and contingentism remains the default position.

Finally, Modality to Essence refers to the canonical reduction to essence presented in Fine's 1994 paper, which is currently one of the most popular candidate reductions of metaphysical modality. ${ }^{3}$ Here's the idea. First, Fine (1994) presents well-known arguments for thinking that essence can't be reduced to familiar modal notions and so we should recognize a non-modal notion of essence (where throughout the paper we'll use 'non-modal' to mean 'not reducible to familiar modal notions'). Taking these arguments on board, he then suggests that we can reverse the standard order of priority by using this nonmodal notion of essence to reduce various modal notions. The nonmodal notion of essence gives us, for any object $o$, a class of propositions essential to $o$; call this class o's essence. For example, Sanders' essence arguably includes the proposition that Sanders is self-identical if Sanders is something, the proposition that Sanders is human if Sanders is something, and so on. We're more used to thinking of objects having certain properties essentially, rather than certain

\footnotetext{
${ }^{3}$ In a recent survey of reductions of metaphysical modality, Cameron (2010) includes the reduction to essence as one of what he regards as the three most promising options, the other two being modal realism and neo-conventionalism. For some authors who have adopted Fine's reduction to essence and continued working on the idea, see Hale (1996), Correia (2006, 2012), Rosen (2006), and Dasgupta (2016). Note that throughout the paper we'll ignore distinctions between different notions of essence and modifications of the original reduction that don't bear on our tension (such as those that appeal to grounding or a notion of consequence relative to some familiar background logic), though we'll discuss potentially relevant modifications later on, including one suggested by Fine's formal work on the reduction to essence. See Fine (1995b) and the works just cited for more notions of essence and some modifications of the reduction that don't bear on our initial tension. Throughout, we're talking about what Fine (1995b) calls 'constitutive immediate essence', which most closely corresponds to the pretheoretical notion.
} 
propositions being essential to objects (which is required for Fine's reduction), but it's not hard to move from one regimentation to the other: Fine can interpret talk of some object $o$ having some property $F$ essentially in terms of the proposition that $o$ is $F$ being essential to $o$. Finally, it's important for Fine's reduction that not only individual objects but also pluralities of objects have essences; that is, propositions can be essential to pluralities of objects in addition to individual objects. For example, Fine might claim that although the proposition that it's not the case that Sanders is identical to Clinton is essential to neither Sanders nor Clinton, the proposition is essential to the plurality consisting of both Sanders and Clinton.

This non-modal notion of essence allows us to define different necessity operators by looking at different objects or pluralities of objects, each of which gives rise to a class of propositions (the propositions essential to the object or plurality of objects). In particular, Fine contends that if we consider the essences of all objects or pluralities of objects whatsoever, the resulting necessity operator will correspond to our familiar notion of metaphysical necessity. So the resulting reduction says that a proposition $p$ is metaphysically necessary in virtue of there being (unrestrictedly) some object or plurality of objects such that $p$ is essential to that object or plurality of objects; we then define metaphysical possibility using the standard dual equivalence (I'll comment on the ideology of 'reduction' and 'in virtue of' shortly). This reduction of modality to essence is committed to the following claim: given any putative metaphysical necessity $p$, there is some object or plurality of objects whose essence contains $p$; in a slogan, all metaphysical necessities originate from the essences of things.

Before we get going, we need some notation, which will simplify our discussion in what follows (note that I'll continue to ignore use-mention distinctions when doing so won't lead to any confusion). We'll use $x x$ and $y y$ throughout the paper as variables ranging over pluralities of objects, where crucially we'll take this to include the special case where the plurality contains just a single object, and we'll use $\sqsubseteq$ for a relation of containment between pluralities, akin to the subset relation (the exact logic of the containment relation won't be important in what follows). Also, throughout, $p$ and $q$ are variables ranging over propositions. We'll follow Fine in using $\square_{x x} p$ to say that $p$ is essential to $x x$ (this isn't exactly Fine's regimentation, but the differences won't matter). So we can state the canonical reduction to essence we just saw, from Fine 1994, as follows: for any $p, \square p$ reduces to 
$\exists x x\left(\square_{x x} p\right)$. I should flag that this formulation of the reduction may at first appear importantly different from Fine's own, which says that a proposition $p$ is metaphysically necessary in virtue of $p$ being essential to the plurality of all objects whatsoever, that is, $\square p$ reduces to $\exists x x \forall y y\left(y y \sqsubseteq x x \wedge \square_{x x} p\right)$. However, Fine takes essence to be monotonic: necessarily, for any $p, x x$, and $y y$, if $\square_{x x} p$ and $x x \sqsubseteq y y$, then $\square_{y y} p$. To get a feel for this assumption, consider the proposition that Sanders is self-identical if Sanders is something, and suppose that the proposition is essential to Sanders. Then given monotonicity, the proposition is also essential to the plurality of Sanders and Clinton, the plurality of Sanders, Clinton and the White House, and also to any other plurality that contains Sanders. More generally, given the monotonicity assumption, any proposition essential to any object or plurality of objects will also be essential to the plurality of all objects whatsoever. As a result, Fine's formulation of the canonical reduction becomes necessarily co-extensive with ours. I started with my formulation because I find it gives a clearer picture of how the canonical reduction to essence works, and doesn't prejudge the issue of monotonicity, but for our purposes we could instead adopt monotonicity and use Fine's formulation, so moving forward I'll switch to Fine's formulation when doing so simplifies the discussion.

\section{The tension}

\subsection{Some preliminaries}

Now that we have our three doctrines and our notation on the table, let's turn to showing why the three doctrines are in tension with one another. I've continually referred to our argument as a 'tension' rather than an inconsistency, because we need to go beyond our three initial doctrines to get things going, but each of these additional steps is hard to resist, so the three doctrines that we started out with will remain the most promising places to try to block our argument. As a quick preview of what's to come, given the canonical reduction to essence, what's necessary or possible reduces to the essences of what objects there are. Given contingentism, possibly it's contingent what objects there are. We'll see shortly that, combining these two claims, it's not hard to show that this possible contingency in what objects there are gives rise to possible contingency in what's necessary or possible. But we'll see that from this result we can derive a contradiction provided metaphysical modality obeys either the modal logic $\mathrm{S}_{4}$ or the modal 
logic B (we'll present two arguments, one that employs the assumption about $\mathrm{S}_{4}$ and another that employs the assumption about B). So something has to give. Let's first spell out the additional doctrines we'll need to make these arguments, and then present the arguments more carefully.

First, our arguments will rely on the following doctrine:

Robustness: If the canonical reduction to essence is true, then it's necessarily true, necessarily necessarily true, and so on for all iterated necessitations of the reduction.

Given $\mathrm{S}_{4}$, robustness is equivalent to the doctrine that the canonical reduction to essence is necessarily true if true at all. So far we've made free use of the ideology of 'reduction', and related ideology like 'in virtue of'. All we need to assume here is that under any way of cashing out this ideology, the reduction to essence purports to tell us what it is for a proposition to be necessary, where we understand this latter demand to imply the modal strength embodied in robustness. These 'What is X?' questions are as old as philosophy itself, and I won't attempt to catalogue the different possible things we might mean by these sorts of questions. Still, I think it's uncontroversial that there's an interesting and prominent reading of questions like 'What is it to be a law of nature?', 'What is it to be a proton?', and, our focus here, 'What is it to be necessary?', according to which adequate answers must be non-contingent, and moreover that this reading is what's standardly at issue in philosophical contexts. Apart from this assumption, our discussion can stay neutral on exactly what ideology is involved in giving a reduction of modality (whether 'grounding', Sider's (2011) 'metaphysical semantics', Dorr's (2016) 'identifications', and so on): robustness remains a plausible doctrine whichever way we go.

Second, our arguments will rely not on contingentism itself, but on the following closely related doctrine:

Standard Contingentism: Some proposition $p$ is possibly both essential to some $x x$ and possibly essential to nothing; that is, $\exists p \vee\left(\exists x x\left(\square_{x x} p\right) \wedge \diamond \neg \exists x x\left(\square_{x x} p\right)\right)$.

Standard contingentism implies contingentism given the plausible assumption that, for any proposition $p$, necessarily if $p$ is essential to some $x x$ then necessarily if everything contained in $x x$ is something then $p$ is essential to $x x$. But the converse fails: contingentism strictly speaking leaves open that although possibly it's contingent what there 
is, this contingency never affects which propositions are essential to some $x x$ or other. Still, most contingentists will also accept standard contingentism, and rightly so. For example, it's generally accepted that given any two objects, it's essential to those objects taken together that it's not the case that the first is identical to the second. So let's assume that it's essential to Sanders taken together with Clinton that it's not the case that Sanders is identical to Clinton (hence necessarily it's not the case that Sanders is identical to Clinton given the reduction to essence). Contingentists will also maintain that each of Sanders and Clinton could have failed to be something (though we could run an analogous example provided the contingentist takes any objects to be only contingently something). So non-standard contingentists must maintain that even if both Sanders and Clinton had failed to be something, there couldn't have failed to be some $x x$ such that it's essential to $x x$ that it's not the case that Sanders is identical to Clinton. Yet it's not clear what this $x x$ could be for contingentists. With some imagination we can conceive of a coherent non-standard contingentist: there could be a contingentist who maintains that although possibly it's contingent what there is (for example, she holds that Sanders and Clinton each could have failed to be something), there is nonetheless some deity, a spaghetti monster perhaps, that is necessarily something, and such that, for any proposition $p$, necessarily if $p$ is essential to some $x x$ then $p$ is also essential to this deity. So for this non-standard contingentist, even if Sanders and Clinton had each failed to be something, the proposition that it's not the case that Sanders is identical to Clinton would nonetheless be essential to this deity. We'll see a more plausible version of non-standard contingentism later on (which appeals to properties that are necessarily something), but the view still won't have much to recommend it. So it's safe to say that most contingentists will also be standard contingentists, holding that possible contingency in what there is gives rise to possible contingency in what's essential to some $x x$ or other, and we'll take the implication from the one doctrine to the other on board for the time being.

\subsection{The tension with S4}

Let's start with the tension with $\mathrm{S}_{4}$. We'll derive an inconsistency from: (i) the doctrine that metaphysical modality obeys the modal logic S4, (ii) the canonical reduction of modality to essence, (iii) standard contingentism (which we're taking for granted all contingentists will want to accept), and (iv) robustness. Note that even though, given our assumptions, all expressions of metaphysical modality are to 
be understood in accord with the canonical reduction to essence, I'll still often use unsubscripted modal operators for readability (though feel free to substitute the canonical reduction to essence stated above for ' $\square$ ' and the reduction's dual for ' $\diamond$ ' throughout).

From standard contingentism, we have that $\exists p \vee\left(\exists x x\left(\square_{x x} p\right) \wedge\right.$ $\left.\diamond \neg \exists x x\left(\square_{x x} p\right)\right)$. So, given the reduction to essence and robustness, we have that $\exists p \diamond(\square p \wedge \diamond \neg \square p)$, i.e. $\exists p \diamond \neg(\square p \rightarrow \square \square p)$. But the modal logic $\mathrm{S}_{4}$ has the rule of necessitation: embodied in the doctrine that metaphysical modality obeys the modal logic $S_{4}$ is not only that what's necessary is necessarily necessary, but that this couldn't have failed to be the case. So we have that $\forall p \square(\square p \rightarrow \square \square p)$, which contradicts what we've just derived.

It's not hard to get a picture for what's going on in this abstract derivation. For instance, contingentists will maintain that Sanders could have failed to be something (this isn't implied by their view of course, but generally contingentists hold much stronger views about what could have failed to be something beyond what contingentism officially implies), or equivalently, given the reduction to essence, that the essences of everything there is leave open that Sanders fails to be something. Let $q$ be the proposition that Sanders is human if Sanders is something. Then $q$ is essential to Sanders, and hence necessary given the reduction to essence. But if Sanders were to fail to be something, then arguably $q$ wouldn't have been essential to anything at all, for reasons we already saw when motivating standard contingentism: if Sanders were to fail to be something then what $x x$ would there have been that's essentially such that Sanders is human if Sanders is something? (Again, we'll consider ways to reject the implication from contingentism to standard contingentism later on.) As a result, given the reduction to essence, $q$ is necessary but possibly not necessary, that is, not necessarily necessary, which contradicts the 4 schema.

\subsection{The tension with $B$}

Turning now to the argument that uses the modal logic B instead of $\mathrm{S}_{4}$, here things are less straightforward. An advantage of the argument from $S_{4}$ is that we were able to stay relatively neutral about what's essential to what. We needed to assume only that essences don't rule out standard contingentism-so roughly, for some proposition $p$, the essences of what there is leave open that (i) $p$ is essential to some $x x$, (ii) the essences of what there is at this possibility leave open that something contained in $x x$ fails to be something, and (iii) $p$ would be essential to nothing if something contained in $x x$ were to fail to be 
something. The tension with $\mathrm{B}$ instead of $\mathrm{S}_{4}$ requires one additional substantive assumption about what's essential to what.

In particular, here's what we need to assume about essence to get the tension with $\mathrm{B}$ going:

$(*) \forall p \square\left(\exists x x\left(\square_{x x} p\right) \rightarrow \square\left(\neg \exists x x\left(\square_{x x} p\right) \rightarrow \neg \exists x x\left(\square_{x x} \diamond p\right)\right)\right)$

This says that there cannot be any $x x$ whose essence contains $\diamond p$ but no $x x$ whose essence contains $p$, provided $p$ is essential to some $x x$ (and that, for any proposition, necessarily this claim obtains). To the extent that I have a grip on such matters, $(*)$ strikes me as the sort of claim that those who go in for the notion of essence should find attractive, though I also recognize some pictures where it turns out false. Note that anyone who accepts $(*)$ would likely accept a much more general doctrine about essence from which $(*)$ follows; for instance, the picture thinking I'll use to motivate $(*)$ below predicts that we should also accept the whole range of principles that arise by substituting any string of modal operators for the possibility operator in the final consequent of $(*)$, and also all iterated necessitations of any of these principles. But I've stated the principle in the particular form that will be relevant for our argument below from B. ${ }^{4}$

\footnotetext{
${ }^{4}$ In fact, the universal claim $(*)$ is stronger than what we need to run an argument against the theorems of B as constraints governing metaphysical modality: we can get a counterexample given only the weaker claim that $(*)$ holds of some proposition that can also witness the truth of standard contingentism. I focus on $(*)$ itself in the main text because visions of essence that predict $(*)$ strike me as the most principled motivations for the weaker claim we actually need, and I suspect most proponents of the canonical reduction will go in for visions of this kind. That being said, the fact that we can run an argument against B using only the weaker claim is dialectically relevant for proponents of the canonical reduction with some non-standard metaphysical commitments. In particular, proponents of the reduction can arguably reject $(*)$ provided they buy into the Chandler-Salmon argument against $\mathrm{S}_{4}$, that is, buy into the flexibility of material origins thesis yet reject any of the standard responses to the argument (see the works cited in footnote 1 for some discussion of this thesis and some responses to the Chandler-Salmon argument). This result would be dialectically awkward if we needed to rely on $(*)$ itself to argue against B, because proponents of the Chandler-Salmon argument against $S_{4}$ are among those for whom the argument against B is especially relevant. I won't go through exactly how to generate an objection to $(*)$ from this package of commitments, but it suffices to point out that this heterodox combination of views doesn't offer a way to reject the weaker claim we could rely on instead. To motivate the weaker claim we must point to a plausible witness of standard contingentism and argue that $(*)$ holds of it. For example, let $p$ be the proposition that it's not the case that Sanders is identical to Clinton, which we saw above is arguably a witness of standard contingentism. If so, we know that $p$ would be essential to nothing if Sanders and Clinton were nothing. Now to motivate the weakened version of $(*)$ we just need to argue that $\diamond p$ would also be essential to nothing if Sanders and Clinton were nothing, which we can do using particular instances of the general
} 
The initial conditional antecedent in $(*)$, restricting our attention to propositions that are essential to some $x x$, is intended to rule out the following sort of counterexamples. Consider the proposition that Sanders stands if Sanders is something: it's essential to nothing (standing is a merely accidental property of Sanders), but someone might claim that Sanders is essentially such that he's a possible stander if he's something, it's 'part of what it is to be him' that he can stand. The more complicated $(*)$ is compatible with this view, so long as necessarily nothing is essentially such that Sanders stands if Sanders is something, because $(*)$ 's initial antecedent ensures that the claim vacuously holds for propositions that aren't possibly essential to some $x x$.

Here's an example of the picture thinking that would support a principle like $(*)$. Some claim that a proposition $p$ is essential to some $x x$ just in case $p$ 'tells us what it is to be $x x$ ' or 'tells us what $x x$ is in its very nature'. (Those who like the ideology of essence generally use these sorts of pre-theoretical glosses to warm us up to the notion; see the citations in footnote 3 for some examples.) Now combine this picture thinking with the further idea that, for any $p$ that is possibly essential to some $x x$ (and so avoiding counterexamples from accidental propositions just mentioned), if there is no $x x$ such that $p$ stands in these special relations to $x x$, i.e. there is no $x x$ such that $p$ tells us 'what it is to be $x x$ ', then there is no $x x$ such that $\nabla p$ stands in these special relations to $x x$. This idea has some plausibility. For instance, if there's no $x x$ such that 'part of what it is to be $x x$ ' is for Sanders to be human if Sanders is something (and so no Sanders), then this idea says there's also no $x x$ such that 'part of what it is to be $x x^{\prime}$ ' is for Sanders to be possibly human if Sanders is something (from now on I'll drop the 'if something' qualifier when it won't be relevant). This claim immediately follows provided any candidate $x x$ with an essence in which we might source the proposition that Sanders is possibly human would also be an $x x$ that's essentially such that Sanders is human, which has some pre-theoretical pull. If we combine the two claims in this paragraph (assuming that if the claims are plausible then they hold non-contingently), we get $(*)$.

The picture thinking in the previous paragraph has some plausibility, but I recognize that there could be other doctrines about how essence behaves according to which $(*)$ comes out false. These would be doctrines according to which some $x x$ that propositions

principles I'll use in the main text to motivate $(*)$ itself. Thanks to Jake Nebel for helpful discussions here. 
like $\diamond p$ are essential to needn't contain any $y y$ that $p$ itself is essential to, nor bear some other relation to any $y y$ that $p$ itself is essential to which implies that $x x$ can't be something unless $y y$ is also something. Returning to our previous example, let $q$ be the proposition that Sanders is human if Sanders is something. Then someone might contend that even if $q$ were essential to nothing (and so Sanders were nothing), $\nabla q$ would still be essential to the concept of essence, which couldn't have failed to be something (recall that we're using $\diamond$ to abbreviate the more complicated statement of metaphysical modality in terms of essence). ${ }^{5}$ Alternatively, someone might contend that the property of being Sanders could be something even if Sanders were nothing, and that at such possibilities $q$ is essential to nothing but $\diamond q$ is still essential to the property of being Sanders. I think these pictures arguably go against some of the pre-theoretical glosses which were supposed to help us understand this ideology of essence to begin with. Against the first, even $\nabla q$ seems still in some sense to be about Sanders, and tell us something about what it is to be him, not about what it is to be the concept of essence. Against the second, although I can sometimes get into a frame of mind where both $q$ and $\diamond q$ are essential to the property of being Sanders (assuming for now that there is such a property that could be something even if Sanders were nothing), I'm not sure what principled motivation inherent in the ideology of essence supports a picture where $\diamond q$ but not also $q$ itself is essential to the property. (The option of saying that both $q$ and $\nabla q$ are essential to the property of being Sanders wouldn't offer a way to reject $(*)$, but points towards a way to defend non-standard contingentism, provided the property is necessarily something, a response we'll discuss later on.)

Despite having just made a tentative case for $(*)$ and against two competing pictures, I don't want to put much weight on these issues either way. Once we move to these complex principles about what's essential to what, I start to lose my grip on the notion of essence, and don't have firm judgements one way or the other. I do think (*) has a lot going for it, but I wouldn't want to lean too heavily on the picture thinking that supports the principle. We'll take $(*)$ on board moving forward, which we need to run our argument from B. Even if a proponent of the reduction to essence could come up with an argument

${ }^{5}$ If we heed Correia's (2006) distinction between 'objectual' and 'generic' essence, we should replace 'the concept of essence' here with 'what it is to be essence', understood as irreducibly second-order, but I'll ignore this distinction throughout because it doesn't affect any of our arguments. 
against $(*)$, we could still fall back on our argument from $\mathrm{S}_{4}$ outlined above. Moreover, the result that proponents of the reduction to essence are committed to denying $(*)$ in order to save B (and hence $\mathrm{S}_{5}$ ) would be an interesting result in its own right. The negation of $(*)$ embodies a substantive constraint on how essence behaves, and I suspect that any picture according to which $(*)$ is false will come with commitments of its own, so denying $(*)$ is certainly more than you might have initially thought was needed in order to adequately implement the canonical reduction to essence.

Taking $(*)$ on board, let's show how the tension arises between our various doctrines. In particular, we'll derive an inconsistency from: (i) (*), (ii) the doctrine that metaphysical modality obeys the modal logic $\mathrm{B}$, (iii) the canonical reduction of modality to essence, (iv) standard contingentism (which we're still taking for granted any contingentist will want to accept), and (v) robustness.

The set-up is similar to that with the 4 schema, but the derivation is a bit more involved. Again, from standard contingentism we have:

(1) $\exists p \vee\left(\exists x x\left(\square_{x x} p\right) \wedge \diamond \neg \exists x x\left(\square_{x x} p\right)\right)$

Combining (1) with $(*)$ and standard modal reasoning (valid even in $\mathrm{K}$ and hence $\mathrm{B})$, we can derive:

(2) $\exists p \vee\left(\exists x x\left(\square_{x x} p\right) \wedge \diamond \neg \exists x x\left(\square_{x x} p\right) \wedge \square\left(\neg \exists x x\left(\square_{x x} p\right) \rightarrow \neg \exists x x\left(\square_{x x} \diamond p\right)\right)\right)$

From (2), again with standard modal reasoning, we can derive:

(3) $\exists p \vee\left(\exists x x\left(\square_{x x} p\right) \wedge \diamond \neg \exists x x\left(\square_{x x} \diamond p\right)\right)$

Finally, from (3), using the canonical reduction of modality to essence and robustness, we have:

(4) $\exists p \vee(\square p \wedge \diamond \neg \square \diamond p)$, i.e. $\exists p \diamond \neg(\square p \rightarrow \square \square \diamond p)$

But it's a theorem of B that necessarily what's necessary is necessarily necessarily possible, so we have that $\forall p \square(\square p \rightarrow \square \square \diamond p)$, which contradicts what we've just derived.

Having spelled out our arguments, let's now consider some possible replies. ${ }^{6}$

\footnotetext{
${ }^{6}$ I should mention briefly that we can run arguments related to the two we presented in this section that start from the temporal analogue of contingentism (that at some time something at some time fails to be something). A proper exploration of the temporal setting would require another paper, but many of the issues we discuss for the modal setting and their
} 


\section{Moving forward}

We've seen that given some attractive additional assumptions, combining contingentism, the canonical reduction of modality to essence, and the doctrine that metaphysical modality is governed by either the modal logic $\mathrm{S}_{4}$ or $\mathrm{B}$, we get a contradiction. The plan for the rest of the paper is to explore various morals we might take away from these results. All of these conclusions will be contested views, and none comes cost-free. Along the way we'll also evaluate various doctrines about the interplay between contingency and essence, try to come up with an attractive non-standard contingentist picture, and develop some alternative reductions of modality to essence. So much of what follows will be of interest even independently of the arguments we just gave.

\subsection{Abandoning one of our initial doctrines}

A concessive reply, mentioned at the outset, would be to just reject one of the doctrines that got our arguments going: (i) contingentism, (ii) the doctrine that metaphysical modality is governed by either the modal logic $\mathrm{S}_{4}$ or $\mathrm{B}$, or (iii) the canonical reduction of modality to essence.

The first option of rejecting contingentism would be to embrace Williamson's necessitism, and accept that necessarily everything is necessarily something. This reply can be used to block our arguments, both of which get going by considering possibilities where something there is fails to be something. For necessitists, necessarily there can be

epicycles pop up again in almost exactly the same form, though of course there are also important disanalogies between the two settings. The main principles we need to get the temporal arguments off the ground are that what's possible is always possible and that what's necessary is always necessary (i.e., nothing impossible ever happens); see Dorr and Goodman (forthcoming) for a defence of these compelling connections between modality and time. Combining these principles with the canonical reduction to essence and the temporal analogue of contingentism, we can derive an inconsistency. I won't rigorously state the temporal arguments here, nor explore the various additional assumptions we need to get the arguments going and possible replies. But very roughly, the temporal arguments try to move from variation in what there is across time, to variation in what's essential to what across time, and finally to variation in what's necessary and possible across time given the canonical reduction to essence, which contradicts the two principles connecting modality and time that we just saw. Still, each of these steps requires additional theses that aren't immediate. Ultimately I find the temporal setting less interesting because, unlike the modal setting we're focusing on, I suspect many would be happy to accept some of the possible responses to the temporal arguments. Most notably, I suspect many who reject necessitism would nonetheless be happy take on its temporal analogue (that always everything is always something), which can be used to block the temporal arguments. 
no variation in what there is. So, provided necessitists adopt the principle that, for any proposition $p$, necessarily if $p$ is essential to some $x x$ then necessarily if everything contained in $x x$ is something then $p$ is essential to $x x$, their view predicts that there cannot be any contingency in what's essential to what (and so that standard contingentism fails), and thus that there cannot be any contingency in what's necessary or possible given the canonical reduction to essence. As a result, necessitists can straightforwardly accept the reduction to essence in its canonical form along with the doctrine that metaphysical modality obeys the modal logic $\mathrm{S}_{5}{ }^{7}$

The second concessive option is to reject the theorems of $\mathrm{S}_{4}$ and B as constraints governing metaphysical modality, and so likely hold that metaphysical modality is governed only by the modal logic $\mathrm{T}$. I suspect that most won't be tempted by this line of response (recall footnote 1), though I acknowledge it as one possible way forward.

A third concessive option would be to keep the theorems of $\mathrm{S}_{5}$ as constraints governing metaphysical modality, and also keep contingentism, and so take our discussion as an argument against the canonical reduction of modality to essence. This reply still leaves open that some modification of the canonical reduction might avoid our arguments. However, the modifications we'll look at later on all face obstacles, so our discussion lends some support to the stronger conclusion that we were mistaken to think essence could somehow be used to reduce metaphysical modality, whether via the canonical reduction or some alternative.

All of these replies in effect concede the thrust of our arguments. Contingentism, and the view that at least the principles embodied in the theorems of $\mathrm{S}_{4}$ or B govern metaphysical modality, are each popular doctrines, so either of the first two concessive replies gives us an interesting result: we'd have shown that proponents of the canonical

\footnotetext{
7 Of course, necessitism doesn't imply the principle in the main text that suffices to block our arguments. For instance, some necessitists may tie what's essential to some $x x$ not to whether $x x$ is something (as we've been assuming throughout) but to whether $x x$ is concrete (where here 'concrete' is a placeholder for whatever property the necessitist takes ordinary judgements about the 'contingency of existence' to be tracking). These necessitists would claim that what's essential to you is a contingent matter, because despite being necessarily something, you're only contingently concrete, and what's essential to you changes at possibilities where you're non-concrete. It's easy to see that this kind of necessitist picture implies standard contingentism, and hence is still vulnerable to our arguments. As a result, necessitists with these commitments who are sympathetic to the canonical reduction to essence must reject $\mathrm{S}_{5}$ or else adopt some other reply to our arguments. I'll set aside this sort of necessitist in the main text, and focus only on necessitist pictures that straightforwardly block our arguments. Thanks to Jeremy Goodman for helpful discussions here.
} 
reduction to essence must either embrace necessitism or else adopt a heterodox view about the logic of metaphysical modality. Also, as mentioned near the outset, the reduction to essence is currently one of the most popular reductions of metaphysical modality, so the strong version of the third option would be a striking result.

\subsection{Non-standard contingentism}

A crucial component of our arguments that proponents of the reduction to essence might try to resist is the implication from contingentism to standard contingentism. We saw some motivation for thinking that any contingentist should also be a standard contingentist, but we acknowledged that the implication isn't immediate, and that it's at least consistent to be a non-standard contingentist (that is, to believe that although possibly it's contingent what there is, this contingency cannot affect which propositions are essential to some $x x$ or other). We parodied this combination of views before with the example of the contingentist who believes that even though possibly it's contingent what there is, there is nonetheless a deity that is necessarily something, and such that, for any proposition $p$, necessarily if $p$ is essential to some $x x$ then $p$ is also essential to this deity. But can we find a more tenable non-standard contingentist view?

Here's the most promising option that I know of, though ultimately I still don't think the view has much to recommend it. This nonstandard contingentist combines her contingentism with higherorder necessitism, the doctrine that necessarily it's non-contingent what properties and propositions there are, and also takes on an unrestricted modal comprehension schema defining what properties there are. The result is that necessarily there is a plenitude of properties that are necessarily something. In particular, necessarily, for anything there is, necessarily that thing's haecceity will be among this plenitude of properties, where for any object $o$, o's haecceity is a property that necessarily something instantiates just in case it's identical to $o$. In a slogan, $o$ 's haecceity is the property of being $o$. (I'm glossing over various formal details in how we move from an unrestricted modal comprehension schema to the doctrine that necessarily for any object necessarily that object's haecceity is something. Our goal is just to present this non-standard contingentist option, so the formal details won't be relevant; those interested should look at Williamson 2013, ch. 6. Also, this non-standard contingentist may adopt the particular doctrine about haecceities without accepting higher-order necessitism or the unrestricted comprehension schema, which 
together imply the doctrine, though the view is more principled when it flows from a general theory of properties rather than being a doctrine peculiar to haecceities.)

So this non-standard contingentist accepts that necessarily everything necessarily has a haecceity. The idea then is to claim that, for any proposition $p$, necessarily if $p$ is essential to some $x x$ then $p$ is also essential to the haecceities of the objects contained in $x x^{8}{ }^{8}$ Provided this non-standard contingentist also adopts the principle that, for any proposition $p$, necessarily if $p$ is essential to some $x x$ then necessarily if everything contained in $x x$ is something then $p$ is essential to $x x$, this picture gives her exactly what she wanted: she can now claim that even though possibly it's contingent what there is, this contingency cannot affect what's essential to some $x x$ or other, because, for any proposition $p$, necessarily if $p$ is essential to some $x x$ then $p$ is also essential to the haecceities of anything contained in $x x$, and these haecceities are necessarily something. Let's return to our example in the argument from $\mathrm{S}_{4}$ to see how the view is working. Again let $q$ be the proposition that Sanders is human if Sanders is something, which for our nonstandard contingentist is essential to both Sanders and his haecceity. For this non-standard contingentist, even if Sanders were to fail to be something, his haecceity would still be something, and so $q$ would still be essential to something, and hence still necessary given the reduction to essence. So our argument against $\mathrm{S}_{4}$ fails, even given contingentism and the canonical version of the reduction to essence. The upshot of this non-standard contingentist picture that appeals to haecceities is that, necessarily, contingency in what there is makes no difference to what's essential to some $x x$ or other, and so our arguments never get off the ground.

I acknowledge this non-standard contingentist option as a potential reply to our arguments. Still, there are two important points to keep in

\footnotetext{
${ }^{8}$ I'm writing as if properties, and so haecceities, are themselves objects (i.e. values of firstorder variables). An attractive nominalist-friendly translation of our discussion is to interpret 'property' talk in terms of irreducible higher-order quantification, and make use of Correia's distinction between objectual and generic essence (see footnotes 2 and 5 for relevant citations), but I don't think this non-standard contingentist can take this route. Her strategy seems to require countenancing mixed-order pluralities, containing both objects and properties (because she claims that propositions are essential to both objects and their haecceities), and it's not obvious how to understand these pluralities if properties aren't also values of first-order variables. So there may be an argument against this non-standard contingentist option starting from a higher-order conception of properties, but making this argument rigorous raises some delicate issues that would take us too far afield, so I focus on other arguments in the main text.
} 
mind. First, some argue that the doctrine that necessarily everything necessarily has a haecceity implies necessitism itself. Roughly, these arguments purport to show that one cannot countenance possibilities where some object's haecceity is something but that object is nothing, because that object must allegedly be something in order for the haecceity to have its unique modal profile. ${ }^{9}$ It's unclear exactly how these arguments are supposed to work, so I don't want to lean on them here, but if something in the vicinity of these arguments succeeds then this non-standard contingentist reply collapses into the reply above that simply embraces necessitism. Second, I'm happy enough if my arguments show that proponents of the reduction to essence are committed to this non-standard contingentist package: this picture where necessarily everything necessarily has a haecceity, combined with the doctrine that, for any $p$, necessarily if $p$ is essential to some $x x$ then $p$ is also essential to the haecceities of what's contained in $x x$, is a very particular metaphysical vision, and not something that anybody has taken to be inherent in the canonical reduction of modality to essence. So if it turns out that this non-standard contingentist picture is ultimately the right way forward in light of our arguments then we'd have a significant result.

\subsection{Abandoning the canonical reduction I: 'mere possibilia'}

Throughout we've been talking about 'the canonical reduction of modality to essence', which is what we presented above and what we argued leads to inconsistency. This canonical reduction is the version of the reduction presented in Fine's 1994 paper, and what's almost universally discussed in the literature under the heading of 'the reduction of modality to essence'. We already mentioned the concessive reply of taking our discussion to tell against the whole project of trying to reduce modality to essence. That reply takes for granted that the canonical reduction is the most promising means of implementing a reduction to essence. So it's worth exploring whether there are other means of reducing modality to essence that might avoid our arguments. We'll look at two such proposals, in this subsection and the next.

I should stress that the canonical reduction from his 1994 paper is arguably not Fine's informed view: in his more formal presentations

\footnotetext{
${ }^{9}$ See Williamson (2013, ch. 6) for these arguments, and also Fine's postscript to Prior and Fine (1977), Adams (1981), McMichael (1983) and Fine (1985) for arguments in a similar spirit pitched against Plantinga's theory that allows for uninstantiated haecceities.
} 
of the reduction to essence, Fine (1995a, 2000) adopts a different version of the reduction, which appeals to what he calls 'possible objects', and can be read as rejecting the canonical version of the reduction usually attributed to him. So arguably Fine himself was aware of the issues that arise for the canonical reduction, and saw the need for some kind of modification.

Here's Fine's informed picture. When determining whether some proposition is essential to some $x x$ or other, we don't look only at what there 'actually is', but rather at 'all possible objects', where this latter class of objects is understood as a proper extension of the former class. (See, for example, Fine (1995a), where he presents a logic for essence, including a system $\mathrm{E}_{5}+$ for when 'the domain is taken to consist of all metaphysically possible objects' (p. 250) and then shows that only the system E5+ allows metaphysical modality on the reduction to essence to obey $\mathrm{S}_{5}$, claiming that 'the strengthened system E5+ yields the correct logic for metaphysical necessity' (p. 267).) The crucial fact here for our purposes is that for Fine necessarily it's noncontingent what 'possible objects' there are. As a result, if this modification of the reduction to essence in terms of 'possible objects' is coherent, it provides a way around our arguments for the same reasons that the necessitist can avoid our arguments, outlined above. Nonetheless, I don't think this modification of the canonical reduction offers an attractive response to our arguments.

First, I used scare quotes when presenting the modification, because I'm sceptical of this ideology of 'possible objects' if understood as more encompassing than every object there is. The 'possible objects' are supposed to include both the 'actual objects' and the 'non-actual objects', but arguably the 'actual' qualifier imposes no restriction on what there is (see Williamson 2013, ch. 1 for a compelling defence of this idea and some sceptical discussion of the traditional actualist versus possibilist debate). If so, then there are no 'non-actual objects', and the 'possible objects' turn out to coincide with the 'actual objects', that is, all of the objects there are. So on the most plausible reading of 'possible objects', Fine's view that necessarily it's non-contingent what 'possible objects' there are amounts to a roundabout way of espousing the necessitist response to our arguments, according to which necessarily it's non-contingent what objects there are. We already know that proponents of the canonical reduction to essence who are willing to adopt necessitism can avoid our arguments; what we're after now are ways to implement a reduction to essence that are available to contingentists. To this end, let's set aside scepticism about the ideology 
and grant that the 'possible objects' might go beyond all of the objects there are; we can argue against this modification of the reduction on its own terms. (To distinguish this reading of 'possible objects' from the reading where the view collapses into necessitism, call the 'possible objects' that aren't among the objects there are the 'merely possible objects'. So contingentist-friendly understandings of this modified reduction purport to avoid our arguments by allowing that propositions can be essential to 'merely possible objects' in addition to whatever objects there are.)

My main worry here is that the reduction to essence is advertised as reducing modality to something inherently non-modal (Fine's nonmodal notion of essence), and indeed the canonical version of the reduction we've been discussing throughout makes good on this promise. By contrast, the version of the reduction that makes use of these 'merely possible objects' straightforwardly fails to meet this ambition, unless somehow this ideology is itself further reduced to something non-modal. I suspect that the reduction to essence would have won few adherents if it also had to appeal to the essences of 'merely possible objects' (indeed the phrase 'possible object' appears nowhere in Fine's original 1994 paper), and that the reduction will ultimately look much less attractive however this ideology is cashed out. To see this, let's look at three accounts of 'merely possible objects'.

One option appeals to Lewis' (1986) modal realism, according to which the 'actual objects' are those located in our Lewis-world (roughly, our spacetime), whereas the 'merely possible objects' are those located in any of the other Lewis-worlds in the modal realist's multiverse. But first, it's hard to imagine anybody willing to take Lewis' modal realism seriously and then still wanting to implement something in the spirit of Fine's reduction: the central motivation for modal realism is that it purports to offer a reduction of metaphysical modality to first-order quantification over Lewis-worlds (or over occupants of these Lewis-worlds plus some counterpart-theoretic apparatus). Second, even granting that talk of 'all the objects there are' is restricted to occupants of our own Lewis-world in ordinary contexts, modal realism falls foul of the collapsing into necessitism worry above, provided we can quantify unrestrictedly when stating theses like necessitism. Finally, Lewis' modal realism is, for good reason, nearly universally rejected, and so the doctrine doesn't offer an attractive modification of the canonical reduction, even if it could somehow be used to implement a reduction to essence that avoids our arguments. (See Williamson 2013, ch. 1 for some recent objections to modal 
realism, and also a detailed discussion of why modal realists are arguably committed to necessitism.)

Views that cash out 'merely possible objects' in terms of some abstract objects like properties, propositions or sets do somewhat better (see Fine 2003 for a survey and critical discussion of some of these 'proxy' reductions); in particular, some claim that we have good reason to believe that there are such abstract objects, independently of any ambition to reduce metaphysical modality to first-order quantification over these objects. Nonetheless, even if a view in this spirit offers a reduction to essence that avoids our arguments (by appealing to the essences of these additional abstract objects), this wouldn't be because these abstract objects are somehow 'merely possible' rather than just being more of what there is, but rather because these objects are necessarily something, and so may offer a way to defend nonstandard contingentism given the canonical reduction to essence and some controversial theses about what's essential to what. So on this understanding of 'merely possible objects', Fine's modified reduction is in fact just the canonical reduction wedded to an ontology that leaves room to defend non-standard contingentism. We've already seen one such package (recall the non-standard contingentist view we just saw that appeals to haecceities that are necessarily something), and points analogous to those made there would apply to similar pictures that use other abstract objects. ${ }^{10}$

As a third potential option, in some of Fine's other writings- see his postscript to Prior and Fine 1977, as well as Fine 1985 and 2003-he provides an 'actualist'-friendly translation procedure to eliminate quantification over 'merely possible objects' using strings of quantifiers and modal operators. For me, this story provides the only coherent account of these 'merely possible objects' that are supposedly distinct from any objects there are; however, the story presupposes that we have in hand some notion of metaphysical modality, which we can then put to work in cashing out talk of 'merely possible objects', and so the story does nothing to make the ideology of 'merely possible objects' any more palatable as something we can take for granted when trying to reduce metaphysical modality. (Indeed, when giving these reductions, Fine subscribes to modalism or primitivism, the doctrine

\footnotetext{
${ }^{10}$ Thanks to both anonymous referees for their suggestions on the issues discussed in the previous two paragraphs, and for offering these potential ways to make good on talk of the essences of 'merely possible objects'.
} 
that metaphysical modality is irreducible; see, for example, Fine 2003, p. 171.)

It's no surprise, then, that it's the canonical version of the reduction to essence, which we've been discussing throughout, that many have wanted to champion. Perhaps the main attraction of Fine's view is that it purports to reduce metaphysical modality using only the nonmodal ideology of essence, without the need to take on baggage like Lewis-worlds or abstract surrogates for 'merely possible objects', including 'merely possible worlds'. We've seen that even granting that some 'possible objects' needn't be among whatever objects there are (which is required if the modification of the canonical reduction that includes the essences of all 'possible objects' is supposed to offer contingentists a way around our arguments), under extant ways to try to cash out this ideology the modified reduction faces significant challenges.

\subsection{Abandoning the canonical reduction II: cumulative essences}

Let's look at another possible modification of the canonical reduction to essence, which I'll call the 'cumulative reduction to essence'. As far as I know, nobody in the literature has suggested a modification of the reduction along these lines. The proposal offers an interesting potential reply to our arguments, yet ultimately still faces some major obstacles. $^{11}$

First, we need some new notation. We'll need more than just a few plural variables, so let's now also use $x x_{0}, x x_{1}, \ldots, y y_{0}, y y_{1}, \ldots$ as variables ranging over pluralities of objects (where, as always, this includes the special case in which the plurality contains only a single object), just as we were using $x x$ and $y y$ up until this point. And we also need a device that lets us form new plural terms from old ones; in particular, where $x x_{0}$ and $x x_{1}$ are any pluralities of objects, let $x x_{0} \sqcup x x_{1}$ be the plurality containing all and only the objects contained in either $x x_{\mathrm{o}}$ or $x x_{1}$.

Here's the idea behind the cumulative reduction. First, recall the canonical reduction to essence (where we'll use Fine's original formulation, mentioned in $\$ 2$, which will simplify the exposition in what

\footnotetext{
${ }^{11}$ The idea behind the cumulative reduction was suggested to me by Kit Fine as a potential reply to my arguments. I make no claim that the particular way I've chosen to develop his initial suggestion is exactly what he had in mind, or that something in the spirit of the cumulative reduction is his preferred reply to my arguments. In writing this subsection, I have benefited greatly from discussions about related issues with Kit Fine, Cian Dorr and Jeremy Goodman.
} 
follows): $\square p$ reduces to $\exists x x \forall y y\left(y y \sqsubseteq x x \wedge \square_{x x} p\right)$. More precisely, given any formula containing any number of metaphysical necessity operators, we apply this procedure on each operator to arrive at a new formula, and then the canonical reduction says that the proposition expressed by the former formula reduces to the proposition expressed by the latter.

On the cumulative reduction, we use a more complicated procedure. Any formula will contain some finite number, possibly zero, of occurrences of $\square$ (I'll assume throughout that $\diamond$ is a metalinguistic abbreviation for $\neg \square \neg$, and so we can just discuss metaphysical necessity operators). We can then associate every occurrence of $\square$ in a formula with some finite number of modal depth: an occurrence of $\square$ has modal depth $n$ just in case the occurrence is in the scope of $n$ other occurrences of $\square$. So, for example, in $\square p \rightarrow \square \square p$ the first and second occurrences of $\square$ have modal depth o, whereas the third occurrence of $\square$ has modal depth 1 . The cumulative reduction to essence says the following: (i) where $\square_{n}$ is an occurrence of $\square$ with modal depth $n, \square_{n} p$ reduces to $\exists x x_{n} \forall y y_{n}\left(y y_{n} \sqsubseteq x x_{n} \wedge \square_{x x_{0} \sqcup x x_{1} \sqcup \ldots \sqcup x x_{n}} p\right)$, (ii) given any formula containing any number of metaphysical necessity operators, we apply this procedure on each operator to arrive at a new formula, and then the cumulative reduction to essence says that the proposition expressed by the former formula reduces to the proposition expressed by the latter.

What is the vision behind this cumulative reduction to essence? The crucial change in this reduction to essence is the extra pluralities of objects we're allowed to look at in formulas with iterated necessity operators when trying to find an essence in which to source $p$. In particular, we can now consider the plurality containing any objects we see in the course of evaluating the formula (I've called the reduction 'cumulative' because the objects we're allowed to look at can only increase with each successive iteration of $\square$ ). Picturesquely, the cumulative reduction allows us to keep track of which objects we've already seen as we move across modal space, and to look back at those objects' essences even if we've moved to possibilities at which those objects fail to be something. Notice that with formulas containing only occurrences of $\square$ with modal depth o, the cumulative reduction to essence doesn't differ from the canonical reduction to essence we've been discussing throughout. So the new reduction comes into its own only when we look at formulas containing modal operators which are themselves in the scope of other modal operators. For 
example, applying the reduction to $\square \square p$ we get: $\exists x x_{0} \forall y y_{0}\left(y y_{0} \sqsubseteq x x_{0} \wedge\right.$ $\left.\square_{x x 0}\left(\exists x x_{1} \forall y y_{1}\left(y y_{1} \sqsubseteq x x_{1} \wedge \square_{x x_{0} \sqcup x x_{1}} p\right)\right)\right)$.

The cumulative reduction to essence avoids both our argument from $\mathrm{S}_{4}$ and our argument from $\mathrm{B}$ above, because when we move to possibilities where something contained in $x x$ fails to be something (because it's consistent with the essences of what there is that something contained in $x x$ fails to be something), we can still consider $x x$ 's essence when determining what's necessary or possible at these possibilities. Another way to put this point is that the cumulative reduction to essence implies non-standard contingentism, which we already know avoids our arguments. So we have here a reduction to essence that lets us keep contingentism, $\mathrm{S}_{5}$, and $(*)$ too.

Though it is an interesting way to modify the reduction to essence, the cumulative reduction is ultimately problematic, and so doesn't offer an attractive response to our arguments. Right away notice that the reduction arguably doesn't answer the question that drives the literature on reductions of modality to begin with, namely, 'what is it to be necessary?'. Extant reductions from the literature take the form 'a proposition is necessary in virtue of $\phi$ ', where ' $\phi$ ' describes some non-modal condition that propositions might satisfy (for example, recall that the canonical reduction to essence says that a proposition is necessary in virtue of being essential to some $x x$ ). By contrast, the cumulative reduction offers a contextual picture, according to which the contribution some particular necessity operator $\square$ makes to the reducing non-modal proposition differs depending on how many other necessity operators embed $\square$ (that is, $\square$ 's modal depth). As a result, the cumulative reduction doesn't allow us to fill in the above schema without first knowing the modal context in which the necessity operator occurs, because the translation of the operator into essence-theoretic terms can change across modal contexts. So what we have in the cumulative reduction is a general procedure for reducing any modal proposition to a non-modal one, rather than a univocal account of what it is to be necessary given in non-modal terms that implies such a procedure, as all other extant reductions of modality provide. However, proponents of the cumulative reduction can justly point out that I haven't given any reason to regard the contextual nature of their story as objectionable. I'm not sure what else there is to say in response, so I currently view the contextual nature of the cumulative reduction as unusual, and certainly not what we expected to find when seeking a reduction of modality, but not as a reason to dismiss the reduction from the get-go. 
A second worry is that proponents of the cumulative reduction must hold that any formulas which express the same modal proposition are mapped by the reduction to formulas which express the same essence-theoretic proposition, or else the cumulative reduction won't give a unique reducing proposition in terms of essence for each modal proposition. Whether this commitment is problematic depends on how fine-grained one takes these propositions to be; we won't explore the wealth of options here, but it's not hard to point to some attractive combinations of views which the commitment rules out. For instance, proponents of the cumulative reduction arguably can't hold that $\square \square p$ expresses the same proposition as $\exists q((q=\square p) \wedge \square q)$, because the latter formula contains only occurrences of $\square$ with modal depth 0 , whereas in the former formula the second occurrence of $\square$ has modal depth 1 (an exception would be proponents of the cumulative reduction with very coarse-grained views according to which the different essence-theoretic formulas that arise from this difference in modal depth nonetheless express the same proposition).

This previous example also points to a more general problem: arguably proponents of the cumulative reduction must privilege certain means of expressing various modal principles over others (roughly those formulas where the modal depth of each operator in the formula matches the 'intuitive depth' of the modality that operator contributes to the proposition expressed). For instance, we would standardly take $\square \square p$ and $\exists q((q=\square p) \wedge \square q)$ to be equally legitimate ways to express that $p$ is necessarily necessary. Yet only $\square \square p$ allows the extra components of the cumulative reduction that help avoid our arguments to kick in (because only it contains an operator with modal depth greater than 0$)$. By contrast, $\exists q((q=\square p) \wedge \square q)$ gets mapped by the cumulative reduction to an essence-theoretic formula that's necessarily equivalent to the formula that $\square \square p$ gets mapped to by the canonical reduction (recall that the cumulative reduction agrees with the canonical reduction when we're dealing only with occurrences of $\square$ that have modal depth 0 , and so agrees with the canonical reduction about $\exists q((q=\square p) \wedge \square q))$. Thus the cumulative reduction has more resources than the canonical reduction to answer our argument from $S_{4}$ only if we somehow privilege formulas like $\square \square p$ as expressions of $p$ being necessarily necessary over formulas like $\exists q((q=\square p) \wedge \square q)$. Generalizing from this example, the cumulative reduction avoids our initial arguments only given certain choices of formulas for expressing various modal principles over other natural choices (picturesquely, we must privilege those formulas whose surface syntax 
mirrors the modal doctrine we're trying to express), because whether the cumulative reduction lets us keep track of which objects we've already seen, and so whether it does any better than the canonical reduction as concerns our arguments, is sensitive to these syntactic distinctions. I don't see how the cumulative reduction can do without this problematic commitment to privileged formulas, given that the reduction of some modal proposition is intimately tied to which formula we take to express that proposition.

We have in the cumulative reduction a general procedure for reducing any modal proposition to a non-modal essence-theoretic proposition. I find the contextual nature of the cumulative reduction problematic, though I've said enough to show only that a contextual reduction isn't what we traditionally had in mind when asking for a reduction of modality. Nonetheless, having to privilege certain means of expressing each modal proposition strikes me as objectionable baggage to take on, and a sign that something has gone wrong. For those sympathetic to reducing modality to essence and less worried about these features, the cumulative reduction may offer an attractive response to our arguments, and indeed an independently interesting modification of the canonical reduction to essence.

\section{Conclusion}

As I said from the outset, my main goal here has been to rigorously bring out the tension between our initial popular doctrines and explore the pros and cons of various morals we might take away from our arguments, rather than point to some reply as the one we should adopt moving forward in light of our results. If forced to go one way or the other, I lean towards either the first or third concessive replies from \$4.1: either embracing necessitism or taking our arguments to vindicate the strong conclusion that we should abandon the general project of trying to reduce metaphysical modality to essence, whether via the canonical reduction to essence or some alternative. I lean towards one of these two options because (i) I'm convinced by the problems I brought out for both modifications of the canonical reduction to essence that we discussed, and I'm not sure how else one might modify the reduction to essence so as to avoid our arguments, (ii) I think non-standard contingentism is unmotivated, and (iii) I take at least the theorems of $\mathrm{B}$, and likely also the theorems of $\mathrm{S}_{4}$, and hence the theorems of $\mathrm{S}_{5}$, as non-negotiable constraints governing 
metaphysical modality (putting aside general reasons to be sceptical of the ideology of metaphysical modality, as we've done throughout). Still, even if I'm wrong about where the balance of considerations is pointing, all of the responses we looked at embody controversial metaphysical theses, so no matter which way we go our arguments will have led to some striking conclusions.

Let me close by courting some controversy. A theme which has been in the background throughout our discussion is that perhaps we don't have as good a grip on Fine's non-modal notion of essence as some have thought. My own view is that the current evidence suggesting we have anything approaching a workable understanding of the notion of essence is thin: the ideology is motivated by a few paradigm cases and picturesque glosses that underdetermine how essence behaves in more interesting cases (the difficulty we saw in saying much informative either way about iterated essentialist claims when discussing $(*)$ being just one symptom of this issue). Of course, I haven't said enough to establish this grandiose claim, but our discussion takes us some way towards it, especially if I'm right about the way forward in light of our results. Reducing metaphysical modality is a central role for the ideology of essence, and one it's called on again and again to play. If we hold this role fixed, then we perhaps gain a lever in forming principled judgements about what's essential to what, by reasoning downwards from independently motivated claims involving metaphysical modality (again assuming that the ideology of metaphysical modality is in good standing, as we've done throughout, though I view this as an open issue). So, by lending some support to the conclusion that we shouldn't think essence has any role to play in reducing metaphysical modality, our discussion calls into question this lever that might have put essence on firmer epistemological footing, and so our discussion takes us at least a little bit towards the sceptical claim about the status of the ideology of essence itself. ${ }^{12}$

\footnotetext{
${ }^{12}$ My greatest debts are to Cian Dorr and Tim Williamson, for extremely helpful comments on earlier drafts of the paper, and for being continual sources of guidance and encouragement. I also owe huge thanks to Andreas Ditter, Kit Fine, Jeremy Goodman, Marko Malink, Jake Nebel, as well as the two anonymous referees and two editors from Mind, all of whom provided comments on earlier drafts of the paper that led to substantial changes. Finally, for helpful comments and discussions about the paper and related issues, thanks to Dave Chalmers, Brian Cutter, Boris Kment, Annina Loets, Adam Lovett, Jonathan Schaffer, and the audience at NYU's 'Washington Square Circle' work in progress seminar.
} 


\section{References}

Adams, Robert Merrihew 1981: 'Actualism and Thisness'. Synthese, 49(1), pp. 3-30.

Cameron, Ross P. 2010: 'The Grounds of Necessity. Philosophy Compass, 5(4), pp. 348-58. doi: 10.1111/j.1747-9991.2010.00296.x.

Chandler, Hugh S. 1976: 'Plantinga and the Contingently Possible'. Analysis, 36(2), pp. 106-9. doi: 10.2307/3327104.

Correia, Fabrice 2006: 'Generic Essence, Objectual Essence, and Modality'. Noûs, 4o(4), pp. 753-67.

2012: 'On the Reduction of Necessity to Essence'. Philosophy and Phenomenological Research, 84(3), pp. 639-53.

Dasgupta, Shamik 2016: 'Metaphysical Rationalism'. Noûs, 50(2), pp. 379-418. doi: 10.1111/nous.12082.

Dorr, Cian 2016: 'To Be F Is To Be G'. Philosophical Perspectives, 30: Metaphysics, pp. 39-134.

MS: Counterparts. Unpublished manuscript.

Dorr, Cian and Jeremy Goodman forthcoming: 'Diamonds Are Forever'. To appear in Noûs.

Fine, Kit 1985: 'Plantinga on the Reduction of Possibilist Discourse'. In James E. Tomberlin and Peter van Inwagen (eds.), Alvin Plantinga, pp. 145-86. Dordrecht: D. Reidel. doi: 10.1007/97894-009-5223-2_4.

1994: 'Essence and Modality'. Philosophical Perspectives, 8, pp. 1-16. doi: 10.2307/2214160.

1995a: 'The Logic of Essence'. Journal of Philosophical Logic, 24(3), pp. 241-73.

- 1995b: 'Senses of Essence'. In Walter Sinnott-Armstrong, Diana Raffman and Nicholas Asher (eds.), Modality, Morality, and Belief: Essays in Honor of Ruth Barcan Marcus, pp. 53-73. Cambridge: Cambridge University Press.

2000: 'Semantics for the Logic of Essence'. Journal of Philosophical Logic, 29(6), pp. 543-84.

2003: 'The Problem of Possibilia'. In Michael J. Loux and Dean W. Zimmerman (eds.), The Oxford Handbook of Metaphysics, pp. 161-79. Oxford: Oxford University Press.

Hale, Bob 1996: 'Absolute Necessities'. Noûs, 30, pp. 93-117. doi: $10.2307 / 2216238$.

Hawthorne, John 2006: 'Determinism De Re'. In his Metaphysical Essays, pp. 239-43. Oxford: Oxford University Press. 
Leslie, Sarah-Jane 2011: 'Essence, Plenitude, and Paradox'. Philosophical Perspectives, 25(1), pp. 277-96.

Lewis, David 1968: 'Counterpart Theory and Quantified Modal Logic'. Journal of Philosophy, 65(5), pp. 113-26. 1986: On the Plurality of Worlds. Oxford: Blackwell.

McMichael, Alan 1983: 'A Problem for Actualism About Possible Worlds'. Philosophical Review, 92(1), pp. 49-66. doi: 10.2307/ 2184521.

Murray, Adam, and Jessica Wilson 2012: 'Relativized Metaphysical Modality'. In Karen Bennett and Dean Zimmerman (eds.), Oxford Studies in Metaphysics, vol. 7, pp. 189-226. Oxford: Oxford University Press.

Prior, A. N., and Kit Fine 1977: Worlds, Times and Selves. London: Duckworth.

Rosen, Gideon 2006: 'The Limits of Contingency'. In Fraser MacBride (ed.), Identity and Modality, pp. 13-39. Oxford: Clarendon Press.

Salmon, Nathan U. 1981: Reference and Essence. Princeton, NJ: Princeton University Press.

1989: 'The Logic of What Might Have Been'. Philosophical Review, 98(1), pp. 3-34. doi: 10.2307/2185369.

Sider, Theodore 2011: Writing the Book of the World. Oxford: Oxford University Press.

Williamson, Timothy 1990: Identity and Discrimination. Oxford: Blackwell.

2013: Modal Logic as Metaphysics. Oxford: Oxford University Press. 